\title{
Hacia una Psicología Social de la Educación
}

SUSANA SEIDMANN, CLARILZA PRADO DE SOUSA (Org.)

\section{BUENOS AIRES: TESEO, 2011, 238 p.}

El libro organizado por Susana Seidmann y Clarilza Prado de Sousa es el resultado de las discusiones originadas en el verano del 2008 en el Seminario Internacional Argentina-Brasil, realizado en Buenos Aires, al que asistieron investigadores de ambos países dedicados al estudio de problemáticas vinculadas a la educación. El objetivo principal del 
seminario fue dar los primeros pasos en la conformación de "una psicología social de la educación”.

Tal como señalan las organizadoras en la introducción al libro, el objeto de estudio de esta nueva subespecialidad estaría constituido por los procesos simbólicos que tienen lugar en los procesos educativos. Los distintos autores reunidos en torno a esta problemática se ocupan, desde diferentes perspectivas, del estudio de los conocimientos de sentido común, en tanto intervienen constitutivamente en los procesos simbólicos que enmarcan y determinan las relaciones entre la enseñanza y el aprendizaje escolar. El aporte central de este trabajo es introducir el análisis de los componentes de la vida cotidiana (creencias, conocimientos, emociones, etc.) en el ámbito escolar, dedicado específicamente a la enseñanza de los conocimientos validados científicamente. Asimismo, estudiar los procesos constitutivos de los sujetos de la educación (educadores y alumnos), requiere ubicarlos en un marco político, que no remite solo a las buenas condiciones de trabajo, sino también a los objetivos y la direccionalidad de su formación.

Esta obra puede leerse de manera lineal, siguiendo el orden de los capítulos, aunque también es posible establecer un recorrido a partir de tres temáticas transversales que, si bien se encuentran presentes en todos los capítulos, adquieren mayor saliencia en algunos de ellos: relevancia de la teoría de las representaciones sociales para el estudio de la identidad docente; articulaciones entre la psicología social y la psicología del desarrollo; propuestas metodológicas para el estudio de los fenómenos educativos.

En el primer capítulo, Clarilza Prado de Sousa y Lúcia Pintor Santiso Villas Bôas analizan la potencialidad del concepto de "representaciones sociales" para estudiar las significaciones colectivas presentes en los procesos educativos. Señalan que, durante la segunda mitad del siglo XX, los estudios desarrollados en el contexto brasileño (y podríamos agregar también en el argentino) han llevado a explicaciones dicotómicas, centradas de manera excluyente en los procesos psicológicos o sociológicos implicados en las situaciones educativas. Justamente, la potencialidad de la teoría de las "representaciones sociales" reside en permitir atrapar los fenómenos psicosociales, ubicados en la intersección entre lo psicológico y lo social. Así, la psicología social introduce en el estudio del campo educativo la pertenencia grupal de los sujetos, en contraposición a los enfoques centrados en el desarrollo de capacidades o características personales, tanto de los docentes como de los alumnos. Dicho de otro modo, la teoría de las representaciones sociales permite estudiar la constitución del sujeto social en el contexto escolar, al postular que la subjetividad es una construcción históricamente situada, que se lleva a cabo en la relación con otros, por ejemplo, un maestro se constituye 
como tal en la relación que establece con sus alumnos y viceversa. Esto último se pone de manifiesto en los hallazgos de las autoras respecto de la representación social hegemónica de la función docente en Argentina, Portugal y Brasil cuyos contenidos refieren a la vocación, a las prácticas de cuidado y a la angustia propia de una actividad cuyo éxito depende del otro.

La psicologización y la sociologización que tuvieron lugar en el estudio de los procesos educativos, también se produjeron en el modo de pensar la identidad docente. Es en este sentido, Vera Maria Nigro de Souza Placco y Vera Lucia Trevisan de Souza, señalan que en los abordajes centrados en procesos psicodinámicos, en la imagen de sí, en la autoestima o en el rol o las actitudes de los docentes, se ha perdido las esencia psicosocial del fenómeno. El recorrido analítico que realizan las autoras pone de manifiesto que la pluralidad de sentidos que se le ha dado al término "identidad" demanda un trabajo particular para los investigadores que se dediquen a su estudio: la elucidación de su polisemia, para poder asumir una perspectiva entre las múltiples posibles de manera reflexiva. Particularmente, en el caso de la identidad docente, su abordaje requiere pensarla en relación dialéctica con los otros agentes de los procesos educativos, especialmente los alumnos, es decir, se vuelve necesario asumir un enfoque psicosocial para su estudio.

En este sentido, Susana Seidmann, Sandra Thomé y Jorgelina Di Iorio, señalan que en las distintas corrientes teóricas que componen en la actualidad la psicología social existe un consenso en torno a pensar la identidad individual como un proceso social, que se lleva a cabo en la relación dialéctica entre el yo, el mundo social y los otros. Específicamente, la teoría de las representaciones sociales, enfatiza la interdependencia social de los individuos, en contraposición al individualismo característico de la denominada "psicología social psicológica”. Las autoras enfatizan la potencialidad del concepto de "representación social” para la comprensión de los fenómenos educativos, en tanto se basa en una ontología dialogal: su punto de partida es la tríada ego/alter/objeto. En los procesos dialógicos las antinomias presentes en el sentido común (themathas) se actualizan convirtiéndose en núcleos de sentido constitutivos de las representaciones sociales. De esta manera, los sujetos de la educación se construyen en relaciones de oposición entre significados y de implicación en los fenómenos dialógicos de la experiencia social. Específicamente, las autoras describen sus hallazgos respecto de las representaciones de los maestros sobre su rol docente, en los que se pone de manifiesto la tensión entre profesionalización/profesión y vocación/profesionalización.

Por su parte, José Antonio Castorina, se ocupa de las bases epistemológicas sobre las que se puede establecer la compatibilidad entre la teoría de las representaciones sociales y el programa de investigación 
de la psicología genética que ha asumido una posición constructivista crítica. Con este objetivo, analiza las relaciones entre ambas disciplinas centrándose en: la concepción del objeto de conocimiento; el tipo de constructivismo asumido y el modo de pensar el cambio representacional. Asimismo, plantea la relevancia de la tesis de la "polifasia cognitiva", propia de la psicología social, para el estudio de los procesos de aprendizaje de las ciencias sociales. Tal como señala el autor, ya no es posible pensar en el desarrollo universal de nociones que progresan hacia estados de mayor validez de los conocimientos, porque no se trata de la construcción de conocimientos por parte de un sujeto solitario, sino que los significados y prácticas sociales de las que los individuos participan restringen sus posibilidades para comprender el mundo, a la vez que las posibilitan. De esta manera, la contribución fundamental de la psicología social para la comprensión de los procesos de aprendizaje es el haber puesto de manifiesto que las identidades sociales de los alumnos intervienen en los procesos que se desarrollan en la sala de clases y haber posicionado a los sujetos de la educación en un contexto social e histórico constituido por sistemas significantes que los anteceden porque forman parte de la memoria colectiva de su grupo de pertenencia.

Asimismo, Abigail Alvarenga Mahoney y Laurinda Ramalho de Almeida analizan las relaciones entre la psicología social y la psicología del desarrollo a partir de la teoría de Henri Wallon. Para este autor, el ser humano es genéticamente social, es decir, el psiquismo humano se transforma constantemente por las relaciones entre los componentes genéticos y los sociales, entre los que se destacan los procesos educativos formales. El sí mismo se desarrolla en función de su identificación con los intereses, objetivos y aspiraciones de su grupo de pertenencia, y a su vez, por la diferenciación necesaria para ocupar un lugar determinado en la conformación social. Por lo tanto, las autoras afirman que la escuela no puede considerar al niño independientemente de su entorno. Señalan que son necesarios análisis que combinen un nivel de análisis sociológico, para comprender el grupo social al que pertenecen los alumnos, con las distintas etapas del desarrollo descriptas por Wallon respecto de las relaciones que los niños de distintas edades establecen con su entorno social. Este autor concebía a la escuela como un campo privilegiado para integrar los polos del individuo y la sociedad en los procesos formativos. Asimismo, la institución escolar abre nuevas posibilidades para el establecimiento de relaciones interpersonales en el tránsito por los distintos grupos que la conforman. El papel de los grupos que integran la institución escolar, y el del profesor como mediador en el aula, podrían llevar a el establecimiento de un medio de desarrollo en el que se construya un ambiente emocional cognitivo y motor favorable para la formación de individuos solidarios y responsables a nivel social, contribuyendo a la generación de mejores condiciones de vida. 
Respecto de las estrategias metodológicas que la psicología social puede aportar para el estudio de los fenómenos educativos, Tarso Mazzotti y Alda Judith Alves-Mazzotti, proponen el análisis retorico un instrumento para la identificación de las representaciones sociales, en tanto éstas se constituyen en contextos de conversación en las que se utilizan argumentos para persuadir a otros. En las diversas interacciones sociales, las personas deliberan sobre las creencias y valores construidas a lo largo de la historia de su grupo, sobre lo que es aceptable o no. Las representaciones sociales se construyen en la negociación de significados que se realiza a partir de las posiciones de los participantes en esas interacciones, de allí la riqueza del análisis retórico para estudiar los "lugares de preferencia o argumentos preferibles" utilizados por los interlocutores, es decir, aquellos esquemas argumentales (de cantidad, cualidad, orden, etc.) que son considerados válidos por los grupos a los que los interlocutores pertenecen, esto es, las razones que alegan para defender lo que consideran preferible hacer o los valores que orientan sus prácticas. Sin duda, la pertinencia de las técnicas de análisis retorico para la investigación de representaciones sociales requiere ser puesta a prueba mediante su utilización en investigaciones empíricas, lo cual llevará a su perfeccionamiento y ajuste a este objeto de estudio para el cual no fueron diseñadas. No obstante, permitirá recontextualizar los enunciados discursivos que, en gran parte de las investigaciones sobre representaciones sociales, son incluidos como una colección de dichos o palabras suficientes en sí mismas para expresar los significados grupales.

Otra estrategia metodológica fructífera para indagar la existencia de representaciones sociales como mediadores simbólicos de los procesos educativos, es la construcción de biografías de formación docente, un método que dentro de las historias de vida se ha consolidado en las ciencias sociales. A este respecto, Marília Claret Geraes Duran y Norinês Panicacci Bahia revisan distintos trabajos que han puesto de manifiesto que gran parte de los recuerdos almacenados en la memoria humana dependen de un proceso de construcción social, asimismo establecen interesantes relaciones con la teoría sociohistórica, en tanto ésta concibe a la memoria humana como el resultado de la internalización de los signos culturales en la interacción con otros. Proponen el uso de la biografía de formación como estrategia para estudiar representaciones sociales, porque permite acceder a una réplica interiorizada de la historia del sujeto al momento de formarse y por ello posibilita aproximarse al proceso de construcción de su identidad docente. Tal como plantean las autoras, recordar narrativamente la propia historia implica un proceso de subjetivación. Esto último implica una ruptura con un enfoque basado en supuestos epistemológicos clásicos que considera a las biografías como estrategias para recolectar información oral, para pensarlas como un 
proceso dialectico en el que se constituye el mismo sujeto al recordar. Así, las autoras afirman que las biografías de formación son fructíferas para la indagación de representaciones sociales porque éstas se constituyen de manera conjunta con las identidades sociales de los individuos. Además, el método biográfico es emancipador, en tanto permite a los adultos, al elaborar sus identidades, criticar las ideologías en las que se encuentran sumergidos, lo cual abre un abanico de posibilidades para su uso en la formación docente.

Finalmente, Claudia Davis y Wanda Maria Junqueira de Aguiar presentan los aportes de la ergonomía francesa como una alternativa para superar la dicotomía entre saber y acción en la investigación y formación docente. Los enfoques tradicionales del trabajo y la formación docente priorizan de manera dicotómica o bien los aprendizajes conceptuales de tipo académico, o bien la formación en las prácticas. Por el contrario, el dispositivo para el análisis de las prácticas profesionales que ha desarrollado la ergonomía francesa permite articular esos dos campos de la formación docente, realizando una reflexión conceptual sobre las prácticas efectivas que tienen lugar en el aula. Desde esta perspectiva, la actividad docente es entendida como "un sistema de actividad", tal como este es descripto por la teoría sociohistórica. Las autoras describen los pasos que requiere este dispositivo de análisis de las prácticas docentes, centrándose particularmente en una de sus herramientas más originales y fructíferas: la autoconfrontación (simple o cruzada). Señalan que este procedimiento permite desplegar un proceso formativo que de lugar a la transformación de las prácticas, a partir de la observación y reflexión de los docentes sobre su propia actividad y la interlocución con sus pares,

Sin duda este libro constituye un aporte fundamental para poner en relieve los aportes de la psicología social para el estudio de los fenómenos educativos. Los vínculos entre los dos campos disciplinares no son obvios, para su comprensión se requiere de trabajos como éste, dedicados al análisis de los conceptos y las metodologías propias de cuerpos teóricos que fueron construidos respecto de problemáticas y niveles de análisis diferentes. E en este sentido, que los distintos trabajos que conforman esta obra pueden ser leídos con un espíritu interdisciplinario, como un prometedor entrecruzamiento de conceptos y modos de investigar entre campos científicos que configuran entramados fructíferos para problematizar los procesos educativos. 